\title{
Deceptively complex diagnosis of early onset primary chronic osteomyelitis: a case report
}

\author{
T. Kavya Priya* (D, Amith Hadhimane, Kirthi Kumar Rai, H. R. Shiva Kumar, Amarnath P. Upasi and \\ Vinayak Gourish Naik
}

\begin{abstract}
Background: Primary chronic osteomyelitis is a rare occurrence in the pediatric mandible though it exclusively affects the mandible with no age preference. The absence of pus, fistula and sequestration are characteristic with insidious onset lacking an acute state. It is a chronic inflammatory disease of unknown etiology. It has two peaks of incidence: initial peak at adolescence and the second peak after the age of 50 years. Clinical and radiological presentation does not suffice the diagnosis of Primary chronic osteomyelitis necessitating biopsy followed by histopathological examination. Use of several terms in the literature to describe this disease entity has led to further confusion. The Zurich classification system satisfactorily describes the early onset Primary Chronic Osteomyelitis based on etiology and pathogenesis.
\end{abstract}

Case presentation: A case of Early Onset Primary Chronic Osteomyelitis in a 10 year old boy is reported comprehensively from clinical presentation to diagnosis and treatment. A meticulous hierarchical order of investigations leads the way to final diagnosis with the aid of existing literature. Extra-oral biopsy, decortication and antibiotic therapy proved to be an effective treatment with no recurrence at 1 year follow-up.

Conclusion: The clinical and radiological features of Early Onset Primary Chronic Osteomyelitis are deceptively complex throwing an array of differential diagnosis including malignant tumors whilst histopathology reveals only chronic inflammation making this entity an enigma. This disease entity should be included in the differential diagnosis for a pediatric posterior mandibular swelling that occurs without an infectious nidus, pus discharge and fistula formation.

Keywords: Primary chronic osteomyelitis, Pediatric mandible, Osteomyelitis of mandible, Early onset, Case report

\section{Background}

Osteomyelitis is one unexampled disease by virtue of presenting varying signs and symptoms. "Primary chronic osteomyelitis is a sterile inflammatory bone disorder of unknown etiology that occurs in pediatric population. The aseptic bone lesions of primary chronic osteomyelitis typically present at a mean age of 10 years as localized pain and swelling, and are histologically characterized

*Correspondence: kavya.priya@icloud.com

Department of Oral and Maxillofacial Surgery, Bapuji Dental College and Hospital, P O Box \#326, Davangere, Karnataka 577004, India by nonspecific osseous inflammation in the absence of infection (Baltensperger and Eyrich 2009). The course of the disease is distinctly variable. Primary chronic osteomyelitis lesions can be acute or chronic, solitary or multifocal, and may resolve, persist or recur" (Baltensperger and Eyrich 2009; Padwa et al. 2016). Umpteen terms are used to describe osteomyelitis of jaws that has given rise to diverse classification systems. The classification systems are based on etiology, pathogenesis, clinical presentation, clinical course, radiology, and histopathology. However, several of these classification systems use more than one criteria confounding and balking comparative 
studies. The sub-classification identifies a significant variant of osteomyelitis of jaws in pediatric population, i.e., early onset primary chronic osteomyelitis. The age of occurrence, history of the disease, clinical presentation and radiological findings lead us to differential diagnosis including vascular lesions, sarcomas and benign tumors. Elimination of each differential diagnosis is a challenging task bearing in mind the postoperative reconstruction options. Early onset primary chronic osteomyelitis should be included in the differential diagnosis of an expansile bony lesion in younger individuals.

\section{Case presentation}

A 10-year-old boy accompanied by his parents reported to the Department of Oral and Maxillofacial Surgery, Bapuji Dental College \& Hospital, with a chief complaint of mild pain and swelling on the right side of lower jaw since a month. Patient noticed that the swelling was gradually increasing in size and pain had always been mild. His medical history revealed off a healthy systemic status. Extra-oral inspection (Fig. 1) showed a diffuse swelling on the right lower third of face of size $3 \times 2 \mathrm{cms}$ extending antero-posteriorly $1 \mathrm{~cm}$ behind the corner of the mouth to angle of mandible, supero-inferiorly $1 \mathrm{~cm}$ above the lower border of the mandible to $1 \mathrm{~cm}$ below the lower border of the mandible. On palpation, the swelling was tender and firm in consistency. Right submandibular lymph node was palpable, of size $0.5 \times 0.5 \mathrm{~cm}$, mobile and non-tender.

Intra oral inspection (Fig. 2) showed mixed dentition with good periodontal status. Buccal cortical bone expansion was evident in the right posterior region from $E$ to 7. Mucosa over the swelling appeared normal. On palpation, the swelling was tender and firm to hard in consistency although a patch of depressed area was evident on the buccal cortical plate which was soft in consistency.

Considering the clinical signs and symptoms, an array of differential diagnosis was laid out. Age of occurrence,

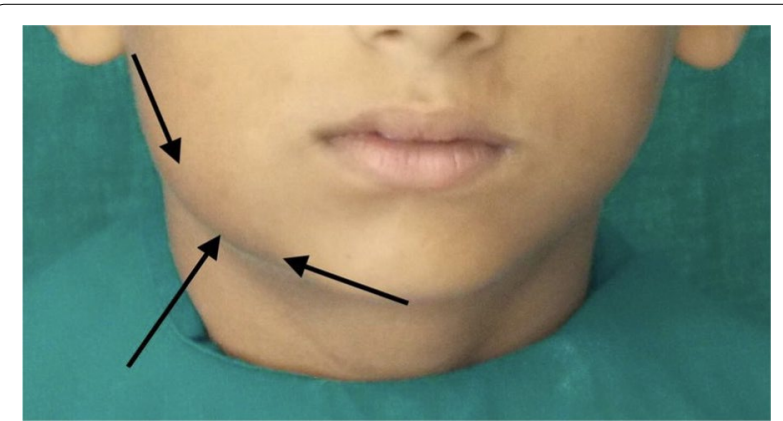

Fig. 1 Extra-oral diffuse swelling on the right lower third of face of size $3 \times 2 \mathrm{cms}$

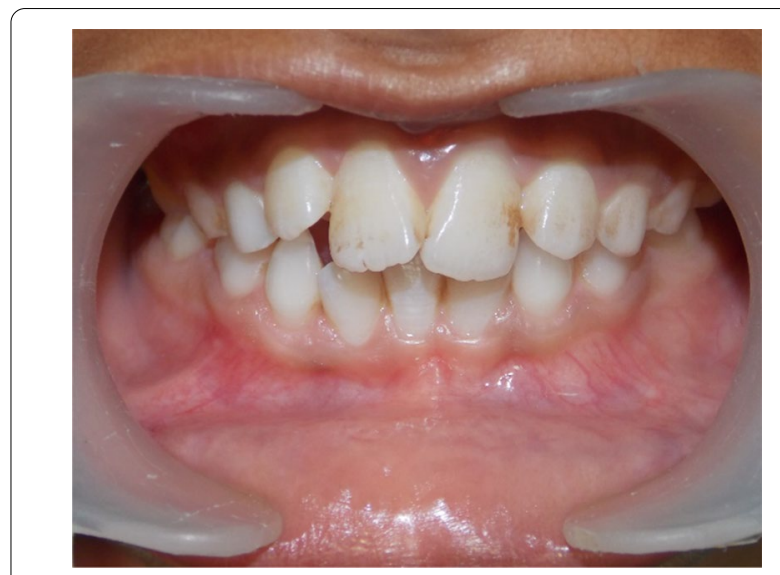

Fig. 2 Mild buccal cortical bone expansion was evident in the right posterior region with respect to $E$

site predilection, common clinical signs and rate of growth suggests Vascular lesion, Ewing's Sarcoma, Juvenile Ossifying Fibroma, Giant Cell Lesion and Ameloblastoma. Further investigations were made to minus each of the differential diagnosis to arrive at the final diagnosis. The patient was subjected to blood and radiographic investigations. A parathormone assay was ordered to rule out Brown tumor and giant cell lesions related to primary hyperparathyroidism. Blood picture including parathormone assay revealed all parameters to be within normal limits. Panoramic radiograph (Fig. 3) showed mixed dentition with appropriate permanent tooth buds. Radiolucency was evident in the region from $\mathrm{E}$ to 7 extending to lower border of mandible with ill-defined and irregular borders.

Patient was further subjected to Ultrasonography to rule out a vascular lesion. USG showed echogenic, expansile lesion within the angle of right side of mandible and also showed few echogenic foci with post acoustic shadowing and internal vascularity suggesting malignant

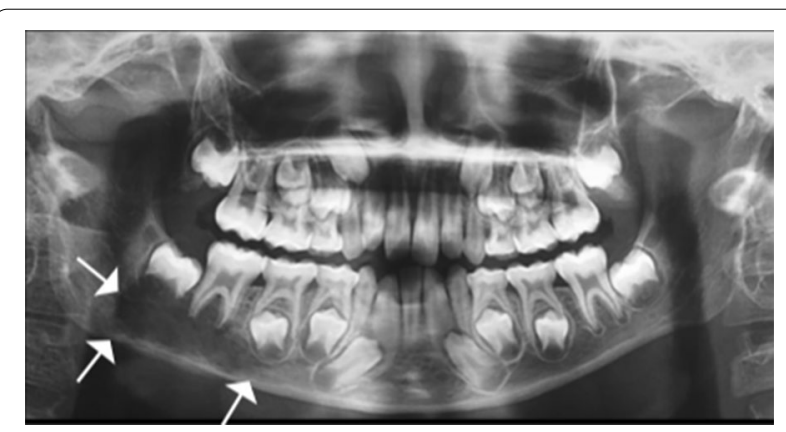

Fig. 3 OPG showing radiolucency in the region from $E$ to 7 extending to lower border of mandible with ill-defined and irregular borders 
lesion within right angle of mandible. So the patient was sent for a contrast enhanced computerized tomography CECT scan to narrow down further. CECT (Fig. 4a, b) showed lytic lesion with expansion of buccal cortex and erosion of outer table at angle of mandible and abnormal enhancing soft tissue within the lytic cavity without showing any periosteal reaction giving an impression of Ameloblastoma.

All the above investigations gave conflicting impressions confounding the diagnosis. It was decided to take the patient for biopsy. Since vascularity and malignancy were suspected there was dilemma regarding the type of biopsy. Incision biopsy was not chosen for two reasons: 1.Excessive bleeding if it is vascular. 2. Traumatizing tissues would aggravate the growth of the tumor if it is malignancy. Submandibular incision was used to access the lesion. Following exposure, buccal cortical erosion was evident, abnormal soft tissue within the lytic cavity was fibrous and had a thick lining adhering to the surrounding bone. Inferior border was intact. The soft tissue was precisely excised (Fig. 5a, b). Extraction of 46 and 47 was done since they were involved within the soft tissue.

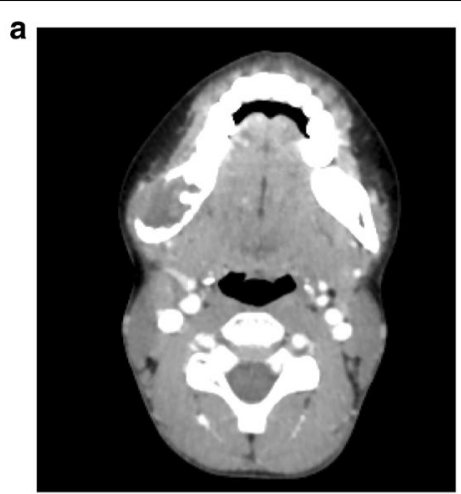

b

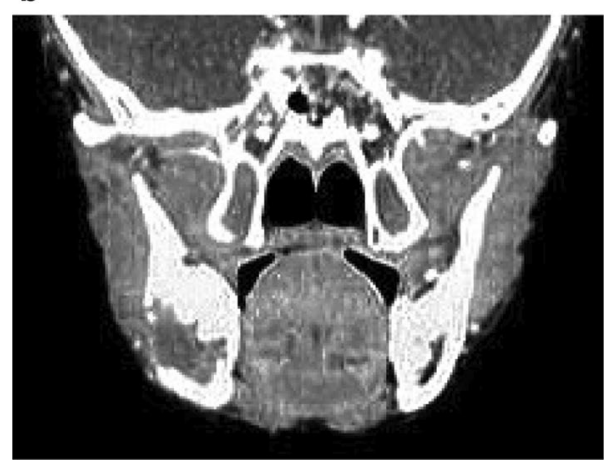

Fig. 4 a Axial CECT image showing lytic lesion with expansion of buccal cortex and erosion of outer table at angle of mandible and abnormal enhancing soft tissue within the lytic cavity without showing any periosteal reaction. $\mathbf{b}$ Coronal CECT image showing the center of the lytic lesion

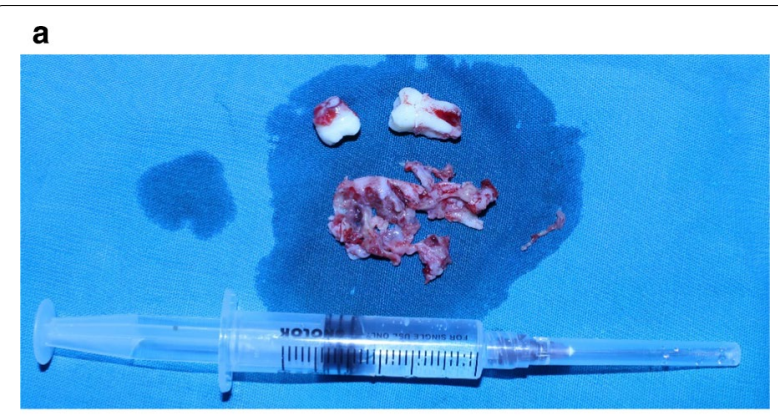

b

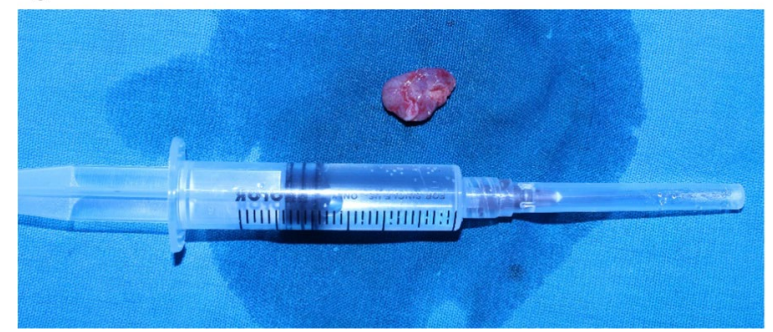

Fig. 5 a The abnormal fibrous soft tissue, mandibular right second molar, incompletely formed mandibular right third molars that were attached to the abnormal fibrous tissue. $\mathbf{b}$ The enlarged right submandibular lymph node was also sent for biopsy

Inferior alveolar nerve was intact and was preserved. Enlarged right submandibular lymph node was excised and sent for biopsy along with the excised lesion. Patient was administered parenteral antibiotics and Non-Steroidal Anti-inflammatory Drugs for pain control for 5 days. He was discharged after 5 days with proper hygiene instructions and was advised a strict follow-up.

Histopathological examination (Fig. 6a, b) showed bone trabeculae, inflammatory infiltrate, mixed inflammation acute and chronic. The medullary space tissue shows fibrosis with granulation tissue formation. The infiltrate consists of macrophages, lymphocytes, plasma cells and a few neutrophilic granulocytes. Lymph node showed only reactive changes. Histopathological examination gives an impression of possibility of chronic osteomyelitis to be excluded in view of acute or chronic inflammation.

After carefully analyzing the clinical data and the subsequent investigations, more so relying on the histopathology we looked up the literature to arrive at a final diagnosis. It was found that the Zurich classification of Osteomyelitis of jaws was the most accepted system. The term "primary chronic osteomyelitis," as used in the Zurich classification of osteomyelitis of the jaws which refers to a rare inflammatory disease of unknown etiology. It is characterized as a strictly non-suppurative chronic inflammation of the jawbone with the absence of pus formation, extra- or intra-oral fistula or sequestration. The absence of these symptoms represents a 

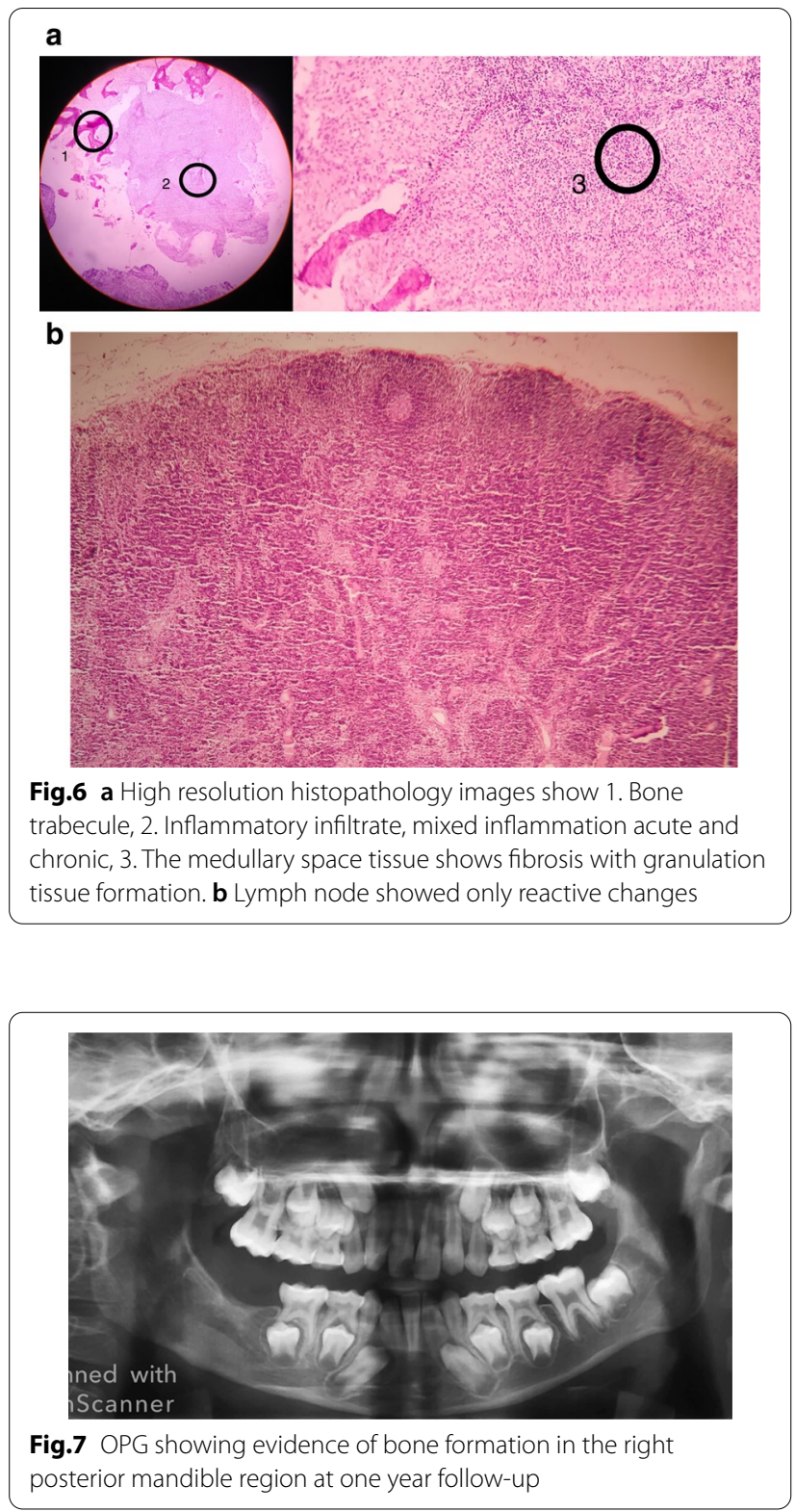

conditio sine qua non and clearly differentiates primary from acute and secondary chronic osteomyelitis in most cases. The term "primary chronic osteomyelitis" also implies that the patient has never undergone an appreciable acute phase and lacks a definitive initiating event. Accordingly, correlating the history, clinical findings, radiological and histology features, a final diagnosis of "early onset primary chronic osteomyelitis of right body of the mandible" was arrived at. The patient was followed up at 1 month, 3 months, 6 months and one year (Fig. 7). The operated site healed uneventfully and there is evidence of good amount of bone formation in the right posterior mandible region at one year follow-up.

\section{Discussion}

"Osteomyelitis" originating from the Greek words, "osteon" meaning bone and "muelinos" meaning marrow is an infection of medullary portion of the bone (Baltensperger and Eyrich 2009). It is defined as a progressive inflammation of the bone and bone marrow (Julien Saint Amand et al. 2017); however, literature feeds numerous definitions to this disease. "Primary chronic osteomyelitis is a sterile inflammatory bone disorder of unknown etiology that occurs in pediatric population. Primary chronic osteomyelitis typically present at a mean age of 10 years as localized pain and swelling, and are histologically characterized by nonspecific osseous inflammation in the absence of infection with highly variable disease course. Primary chronic osteomyelitis lesions can be acute or chronic, solitary or multifocal, and may resolve, persist or recur." (Baltensperger and Eyrich 2009; Padwa et al. 2016). Very less is reported in literature on early onset primary chronic osteomyelitis. Most of the articles and reports are on osteomyelitis in adults. Earlier classifications did not consider osteomyelitis to be age-specific. In a retrospective study of 30 cases of primary chronic osteomyelitis that was conducted by Mark highlighted the age prevalence and found that the age at onset of symptoms demonstrated two peaks of incidence, one before the age of 20 years and one after the age of 50 years (Baltensperger and Eyrich 2009). Prime clinical symptoms were pain, swelling and limited mouth opening. Regional lymphadenopathy was noticed infrequently. These symptoms were vehement in younger patients and presented early on in the course of the disease. The symptoms occur as periodic episodes with pain fluctuating in intensity persisting over a few days to many weeks and also exhibiting a symptom free period (Baltensperger et al. 2004). Vincent's symptom may often be positive in active periods. Absence of pus formation, extra- and/or intra-oral fistula formation and sequestra formation are characteristic features noted (Baltensperger et al. 2004; Eyrich et al. 2007). However, our case did present with all the above clinical features except for Vincent's symptom and restricted mouth opening not making them characteristic features of Primary chronic osteomyelitis. Baltensperger and Eyrich (2009) also mentioned that clinical symptoms were never too disturbing to the patient. This deceptive nature of the disease leads to differential diagnosis such as fibro-osseous lesions and malignancy, viz. Ewing's sarcoma, especially in children and adolescents with significant mandibular enlargement. Two thirds patients in Mark's study markedly had fine oral health with no evidence of odontogenic infection similar to our case (Baltensperger et al. 2004). Julien et al. (2017) in their study found $20 \%$ of the patients had no presumed cause and $80 \%$ had an unclear etiology. Marc et al. 
negated the theory of infection as a cause and said that the dental infection should thus be considered as a confounding factor in Primary chronic osteomyelitis. Bevin et al. found that bacteria isolated from microbiology samples greatly belonged to the oral flora, probably due to contamination unconvincing the infectious etiology of primary chronic osteomyelitis (Bevin et al. 2008; Marx et al. 1994). The various theories on etiologies of primary chronic osteomyelitis also include vascular deficiency, auto-immune disease, immunologic reaction to bacterial toxins, hyperactive immunologic response and muscular hyperactivity (Bevin et al. 2008; Merkesteyn et al. 1990). Nonetheless, clear understanding is not established for any of the theories.

Radiological presentation of primary chronic osteomyelitis reported in literature consists of osteosclerosis as a notable feature though it was not seen in our case. Marc et al. found that sclerosis was primarily seen in adult patients and in advanced stages of early onset cases, whereas a mixed pattern appearance of sclerosis and osteolysis was primarily seen in younger patients and in the early stages of the disease (Baltensperger et al. 2004). However, Goaz and Wood (1985) suggest that primary osteomyelitis can show only as radiolucency on the radiograph in the active stages consistent with our present case. Periosteal reaction and thickening of the mandible observed in patients younger than 20 years appeared as pseudo-tumor impersonating malignancy necessitating biopsy to obtain a definitive diagnosis. However, biopsy is largely considered substantial to diagnose Primary chronic osteomyelitis and to eliminate benign or malignant tumors (Renapurkar et al. 2016). Literature reports various other investigations conducted that include computed tomography scan and bone scintigraphy (Julien Saint Amand et al. 2017). While computed tomography scans showed no evidence of collection, fistula formation or bone sequestration in primary chronic osteomyelitis cases, bone scintigraphy showed significant uptake in the affected areas.

Hjorting-Hansen made a detailed study of the pathology of the gross specimen following surgical decortication. His findings include irregular reactive sub-periosteal bone, thickened cortical plate, infrequent small cavities filled with granulation tissue (Hjørting-Hansen 1970). Similarly, in our study there was evidence of buccal cortical perforation with cavity formation filled with granulation tissue. Microscopic findings in most of the studies present marked chronic inflammation signs like infiltration of macrophages, lymphocytes, plasma cells, neutrophilic granulocytes and some amount of medullary fibrosis and major soft tissue inflammation similar to our present case (Baltensperger et al. 2004; Merkesteyn et al. 1990; Jacobsson and Heyden 1977; Panders and Hadders
1970). However, studies also found micro-abscesses, hyalinosis around small vessels, bone resorption and apposition which were not evident in our case (Eyrich et al. 2003).

Literature consists of several classifications and sub-classifications for osteomyelitis based on etiology, clinical features and radiological features. Further many terminologies are used to describe one type of osteomyelitis leading to confusion. Marc mentions that review of their own patient data regarding primary chronic osteomyelitis were at odds pointing out the confounding classifications in the literature and how terminologies were erroneously used (Baltensperger and Eyrich 2009). In his study, cases that were described as diffuse sclerosing osteomyelitis were later changed to primary chronic osteomyelitis after careful review and cases which showed pus formation and presence of a fistula were changed to secondary chronic osteomyelitis (Baltensperger and Eyrich 2009). Analogously, Groot et al. (1992) also described cases in his study which presented with suppuration and fistula formation as true diffuse sclerosing osteomyelitis. Other authors also spuriously used the terms diffuse sclerosing osteomyelitis and primary chronic osteomyelitis (Hardt and Grau 1987). Marc mentions that since the disease changes its course at certain point of time also changing the presenting symptoms, distinguishing the disease as primary and secondary becomes more difficult. Diffuse sclerosing osteomyelitis solely describes radiological appearance which can be caused by other entities like secondary chronic osteomyelitis, chronic tendoperiostitis and Garre's osteomyelitis/ossifying periostitis on diagnostic imaging (Merkesteyn et al. 1990; Hjørting-Hansen 1970; Groot et al. 1992; Ellis et al. 1977; Eisenbud et al. 1981). To avoid confusion and to rectify the use of erroneous terms, Marc formulated certain criteria to classify types of osteomyelitis and assigned appropriate terms based on these criteria. The most accepted and widely used Zurich classification system described by E. Hjorting-Hanson and then refined by Marx and Mercuri is based on three criteria (Baltensperger and Eyrich 2009). The first criteria are based on clinical appearance, course of disease and radiology which majorly classifies the disease into acute, primary and secondary osteomyelitis. The second criteria are based on gross pathology and histology which helps in excluding differential diagnosis in borderline cases. The third criteria are based on etiology and pathogenesis which further divides the major groups of acute osteomyelitis, secondary chronic osteomyelitis and primary chronic osteomyelitis into subgroups. Marc in his study found that the onset of the disease revealed two incidence peaks, an initial 
incidence peak was noted in adolescence between 11 and 20 years and a second peak after age 50 years. A closer analysis of data further revealed differences in clinical appearance, course as well as in radiology and histopathology of these cases depending on the age of onset of the disease. Based on these differences the established major classification group, primary chronic osteomyelitis, was recently sub-classified into early and adult onset primary chronic osteomyelitis (Baltensperger and Eyrich 2009). Accordingly, a final diagnosis of early onset primary chronic osteomyelitis was assigned for our present case.

Literature delineates two modalities of treatment: medical and surgical. Renapurkar et al. (2016) successfully applied a protocol consisting of extra-oral biopsy, decortication, long-term antibiotic therapy and follow-up with a cure rate of $58.3 \%$. They also found that decortication at an early stage in the disease was efficacious. Similarly, Erych et al. (2003) had a success rate of $54.5 \%$ by treating their patients with biopsy, decortication and antibiotic therapy. Jacobsson and Heyden (1977) and Montonen et al. (1969) in their respective studies successfully treated patients with few benefiting only with long-term antibiotic therapy while some needed decortication to improve the symptoms. Heggie et al. followed a protocol consisting of decortication with Indomethacin without administering antibiotics with a mean follow-up of 3 years while all his patients recovered with the use of indomethacin following surgical intervention (Heggie et al. 2003). Literature also reports the use of adjunctive measures that include Hyperbaric Oxygen therapy and use of steroids (Lentrodt et al. 2007; Obel et al. 2013). Lentrodt in his study reports that administering Hyperbaric Oxygen therapy following decortication in his patients proved beneficial and improved cure rates. Although conventional microbiologic techniques fail to recover organisms in most cases and intra-oral biopsies often result in contamination and inaccurate cultures suggesting a non-infectious disease process, the radiologic, histopathological characteristics and the clinical response to antibiotic therapy support the hypothesis that this is a bone infection (Baltensperger et al. 2004; Renapurkar et al. 2016). The protocol combining extra-oral biopsy, decortication, long-term antibiotic therapy and followup has proven successful in managing this condition. In our study, the same protocol was used successfully with no recurrence and also showed evidence of bone formation at 1 year follow-up. Following treatment, sclerosis becomes more evident as the cortical bone structure undergoes normalization (Baltensperger et al. 2004). Studies by Van Merkesteyn et al. (1990) and Panders and Hadders (1970) support the above findings that it takes more time to find the improvement on radiographs with disease that has substantially affected the structure of the bone.

\section{Conclusion}

Early onset primary chronic osteomyelitis is a rare entity. The clinical and radiological features are deceptively complex throwing an array of differential diagnosis including malignant tumors whilst histopathology reveals only chronic inflammation making this entity an enigma. Meticulous subtraction is necessary to arrive at a diagnosis and an added burden to this is the confusion that exists with the terminology. Primary chronic osteomyelitis should be included in the differential diagnosis for a pediatric posterior mandibular swelling that occurs without an infectious nidus, pus discharge and fistula formation.

\section{Acknowledgements \\ Sincere thanks to Dr.Chatura Kasimsetty, Professor in Department of General Pathology, JJM Medical College, Davangere, Karnataka, for providing the histopathology images for the above case report.}

\section{Authors' contributions}

"All the authors have read and approved the manuscript". Author 1-Dr. KPT-has made substantial contributions to conception and design, acquisition of data, analysis and interpretation of data and involved in drafting the manuscript. Author 2-Dr. AH-was involved in drafting the manuscript and in revising the case report critically for important intellectual content. Author 3-Dr. KKR - has given final approval of the version to be published. Author 4-Dr. SHR — was involved in revising the case report critically for important intellectual content. Author 5-Dr. APU—was involved in revising the case report critically for important intellectual content. Author 6-Dr. VGN-was involved in revising the case report critically for important intellectual content.

\section{Funding}

None.

Availability of data and materials

Data supporting the findings of this Case report are available within the article.

\section{Declarations}

Ethics approval and consent to participate Not applicable.

\section{Consent for publication}

Written informed consent to publish this information was obtained from the parent [Father] of the patient.

\section{Competing interest}

None Declared.

Received: 6 January 2021 Accepted: 8 March 2021

Published online: 19 March 2021

\section{References}

Baltensperger MM, Eyrich GKH (2009) Osteomyelitis of the jaws. Springer, Berlin, pp 1-315

Baltensperger M, Grätz K, Bruder E, Lebeda R, Makek M, Eyrich G (2004) Is primary chronic osteomyelitis a uniform disease? Proposal of a classification 
based on a retrospective analysis of patients treated in the past 30 years. J Craniomaxillofac Surg 32(1):43-50

Bevin C, Inwards C, Keller E (2008) Surgical management of primary chronic osteomyelitis: a long-term retrospective analysis. J Maxillofac Oral Surg 66(10):2073-2085

Eisenbud L, Miller J, Roberts I (1981) Garré's proliferative periostitis occurring simultaneously in four quadrants of the jaws. Oral Surg Oral Med Oral Pathol 51(2):172-178

Ellis D, Winslow J, Indovina A (1977) Garré's osteomyelitis of the mandible. Oral Surg Oral Med Oral Pathol 44(2):183-189

Eyrich G, Baltensperger M, Bruder E, Graetz K (2003) Primary chronic osteomyelitis in childhood and adolescence: a retrospective analysis of 11 cases and review of the literature. J Maxillofac Oral Surg 61(5):561-573

Eyrich G, Harder C, Sailer H, Langenegger T, Bruder E, Michel B (2007) Primary chronic osteomyelitis associated with synovitis, acne, pustulosis, hyperostosis and osteitis (SAPHO syndrome). J Oral Pathol Med 28(10):456-464

Goaz PW, Wood NK (1985) Differential diagnosis of oral lesions: Solitary radiolucencies with ragged and poorly defined borders, 3rd edn. Mosby, pp 440-470

Groot R, van Merkesteyn J, van Soest J, Bras J (1992) Diffuse sclerosing osteomyelitis (chronic tendoperiostitis) of the mandible. Oral Surg Oral Med Oral Pathol 74(5):557-560

Hardt N, Grau H (1987) Der chirurgische Fall. Primär chronische Osteomyelitis des Unterkiefers [Surgical case. 2. Primary chronic osteomyelitis of the mandible]. Schweiz Monatsschr Zahnmed 97(3):353-354

Heggie AA, Shand JM, Aldred MJ, Talacko AA (2003) Juvenile mandibular chronic osteomyelitis: a distinct clinical entity. Int J Oral Maxillofac Surg 32(5):459-468

Hjørting-Hansen E (1970) Decortication in treatment of osteomyelitis of the mandible. Oral Surg Oral Med Oral Pathol 29(5):641-655

Jacobsson S, Heyden G (1977) Chronic sclerosing osteomyelitis of the mandible. Oral Surg Oral Med Oral Pathol 43(3):357-364

JulienSaintAmand M, Sigaux N, Gleizal A, Bouletreau P, Breton P (2017) Chronic osteomyelitis of the mandible: a comparative study of 10 cases with primary chronic osteomyelitis and 12 cases with secondary chronic osteomyelitis. J Stomatol Oral Maxillofac Surg. 118(6):342-348

Lentrodt S, Lentrodt J, Kübler N, Mödder U (2007) Hyperbaric oxygen for adjuvant therapy for chronically recurrent mandibular osteomyelitis in childhood and adolescence. J Oral Maxillofac Surg 65(2):186-191

Marx RE, Carlson ER, Smith BR, Toraya N (1994) Isolation of Actinomyces species and Eikenella corrodens from patients with chronic diffuse sclerosing osteomyelitis. J Oral Maxillofac Surg 52(1):26-34

Montonen M, lizuka T, Hallikainen D, Lindqvist C (1993) Decortication in the treatment of diffuse sclerosing osteomyelitis of the mandible. Retrospective analysis of 41 cases between 1969 and 1990. Oral Surg Oral Med Oral Pathol 75(1):5-11

Obel G, Krogdahl A, Thygesen T, Godballe C (2013) Juvenile mandibular chronic osteomyelitis: 3 cases and a literature review. J Oral Maxillofac Surg 71(2):305-309

Padwa B, Dentino K, Robson C, Woo S, Kurek K, Resnick C (2016) Pediatric chronic nonbacterial osteomyelitis of the jaw: clinical, radiographic, and histopathologic features. J Maxillofac Oral Surg 74(12):2393-2402

Panders A, Hadders H (1970) Chronic sclerosing inflammations of the jaw. Oral Surg Oral Med Oral Pathol 30(3):396-412

Renapurkar S, Pasternack M, Nielsen G, Kaban L (2016) Juvenile mandibular chronic osteomyelitis: role of surgical debridement and antibiotics. J Maxillofac Oral Surg 74(7):1368-1382

van Merkesteyn J, Groot R, Bras J, McCarroll R, Bakker D (1990) Diffuse sclerosing osteomyelitis of the mandible: a new concept of its etiology. J Oral Maxillofac Surg Med Pathol 70(4):414-419

\section{Publisher's Note}

Springer Nature remains neutral with regard to jurisdictional claims in published maps and institutional affiliations.

\section{Submit your manuscript to a SpringerOpen ${ }^{\circ}$ journal and benefit from:}

- Convenient online submission

- Rigorous peer review

- Open access: articles freely available online

- High visibility within the field

- Retaining the copyright to your article

Submit your next manuscript at $\boldsymbol{\nabla}$ springeropen.com 